> Les paragangliomes et les phéochromocytomes sont des tumeurs rares qui se développent aux dépens des ganglions sympathiques et parasympathiques, ainsi que de la médullosurrénale. La génétique des phéochromocytomes et des paragangliomes a fait des progrès considérables au cours des dix dernières années. Dix gènes de prédisposition (VHL, RET, NFI, SDHA, B, C et $D$, SDHAF2, TMEM127 et finalement MAX) ont été identifiés, expliquant plus d'un tiers des cas (avec une histoire familiale, mais aussi de présentation apparemment sporadique). Les études transcriptomiques ont par ailleurs conduit à l'identification de mutations somatiques des gènes RET et VHL dans les tumeurs d'environ $15 \%$ des patients atteints d'une forme sporadique de la maladie, apportant ainsi une explication moléculaire dans près de la moitié des cas. Ces découvertes ont conduit à une meilleure compréhension des mécanismes moléculaires de tumorigenèse des phéochromocytomes et des paragangliomes. II a été montré que le statut génétique des tumeurs était associé à une signature moléculaire particulière, telle que la pseudohypoxie ou l'activation des voies des MAPK ou de mTOR. Ces connaissances ont eu un impact majeur sur le conseil génétique des patients et de leurs familles et permettront à l'avenir une prise en charge médicale, clinique et thérapeutique personnalisée des patients atteints. <

Les paragangliomes (PGL) sont des tumeurs neuroendocrines rares qui se développent aux dépens du tissu paraganglionnaire. Celui-ci est issu de la migration des cellules de la crête neurale et constitue le système nerveux sympathique et parasympathique. Les PGL se développent dans la tête (glomus tympanique

\section{La génétique des paragangliomes et des phéochromocytomes}

Judith Favier ${ }^{1,2}$, Anne-Paule Gimenez-Roqueplo ${ }^{1,2,3}$

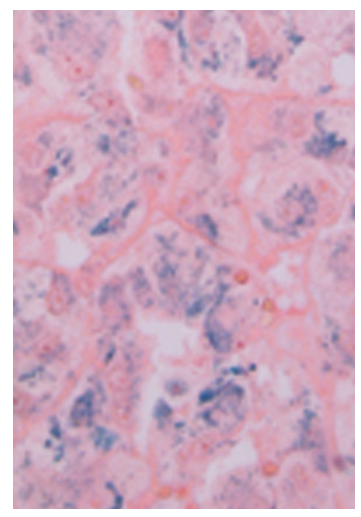

${ }^{1}$ Inserm, UMR 970, Paris-Centre de recherche cardiovasculaire (PARCC) de l'hôpital européen Georges Pompidou, 56, rue Leblanc, 75015 Paris, France ;

${ }^{2}$ université Paris-Descartes, faculté de médecine, Paris, France ;

${ }^{3}$ Assistance publique-hôpitaux de Paris, hôpital européen Georges Pompidou, service de génétique, 56, rue Leblanc, 75015 Paris, France.

et jugulaire), le cou (glomus carotijudith.favier@inserm.fr dien et vagal), la médullosurrénale et les ganglions sympathiques thoraco-abdominaux-pelviens. Ces tumeurs peuvent sécréter des catécholamines; on parle de phéochromocytome (PCC) si leur localisation est médullosurrénalienne, ou de PGL fonctionnel si leur localisation est extrasurrénalienne. Elles constituent alors une cause classique de forme secondaire et grave d'hypertension artérielle. Leur prévalence est évaluée à environ $0,1 \%$ des patients hypertendus et $4 \%$ des patients présentant une masse surrénalienne découverte de façon fortuite. Les PGL/ PCC sont des tumeurs très vascularisées, le plus souvent bénignes. Cependant, elles sont malignes dans environ $15 \%$ des cas et, en absence de critère histologique fiable, ce diagnostic de malignité des PGL/PCC repose sur l'apparition d'une métastase à distance d'un site paraganglionnaire (ganglions, os, foie, poumons, etc.). La seule approche thérapeutique efficace est l'ablation chirurgicale de la tumeur, qui peut être à l'origine de nombreuses complications, notamment du fait de la sécrétion hormonale et de l'importante vascularisation de ces tumeurs. Les PGL/PCC posent donc de difficiles problèmes de diagnostic, de pronostic, de traitement et de surveillance à long terme [1].

Au cours des dix dernières années, nous avons assisté à une progression spectaculaire des connaissances sur la génétique des PGL/PCC. On a longtemps considéré que seules $10 \%$ de ces tumeurs étaient génétiquement déterminées, causées par des mutations constitutionnelles des gènes NFI (neurofibromin) [2], RET (rearranged during transfection) [3] ou VHL (von Hippel Lindau) [4]. Après l'identification des gènes SDHD (succinate dehydrogenase complex, subunit D) [5], SDHC [6] et SDHB [7] dans les années 2000-2001, il apparut qu'au moins un quart des PGL/PCC étaient en fait héréditaires. En 


\begin{tabular}{|c|c|c|c|}
\hline Gène & $\begin{array}{l}\text { Localisation } \\
\text { chromosomique }\end{array}$ & Année & Caractéristiques phénotypiques principales \\
\hline NFI & $17 q 11.2$ & 1990 & PCC isolés, parfois malins \\
\hline RET & $10 q 11.2$ & 1993 & $\begin{array}{l}\text { PCC isolés ou bilatéraux, très rarement malins } \\
\text { Jeune âge au diagnostic }\end{array}$ \\
\hline VHL & $3 p 25.3$ & 1993 & $\begin{array}{l}\text { PCC bilatéraux ou isolés > PGL TAP »> PGL de la tête et du cou, parfois malins } \\
\text { Jeune âge au diagnostic }\end{array}$ \\
\hline$S D H D$ & $11 q 23$ & 2000 & $\begin{array}{l}\text { PGL multiples. PGL de la tête et du cou > PGL TAP > PCC isolés ou bilatéraux } \\
\text { Transmission paternelle }\end{array}$ \\
\hline SDHC & $\operatorname{lq} 23.3$ & 2000 & $\begin{array}{l}\text { PGL de la tête et du cou > PGL TAP } \\
\text { Très rarement malins }\end{array}$ \\
\hline$S D H B$ & $1 p 36.1-p 35$ & 2001 & $\begin{array}{l}\text { PGL TAP > PGL de la tête et du cou = PCC. PGL multiples } \\
\text { Très souvent malins }\end{array}$ \\
\hline SDHAF2 & $11 q 12.2$ & 2009 & $\begin{array}{l}\text { PGL de la tête et du cou. PGL multiples } \\
\text { Transmission paternelle }\end{array}$ \\
\hline TMEM127 & $2 q 11.2$ & 2010 & $\mathrm{PCC}$ isolés $>\mathrm{PCC}$ bilatéraux \\
\hline SDHA & $5 p 15$ & 2010 & PGL de la tête et du cou et PGL TAP \\
\hline MAX & $14 q 23$ & 2011 & PCC isolés ou bilatéraux \\
\hline
\end{tabular}

Tableau I. Les dix gènes de prédisposition au PGL/PCC: localisation chromosomique, année d'identification et principales corrélations phénotypiques. PCC : phéochromocytome ; PGL : paragangliome ; TAP : thoraco-abdomino-pelvien.

2012, dix gènes de susceptibilité au PGL/PCC sont connus (Tableau I) et expliquent près de la moitié des cas.

Depuis plusieurs années, des équipes du monde entier ont étudié de grandes cohortes de patients atteints de PGL/PCC dont I'ADN leucocytaire et les tissus tumoraux avaient été collectés dans des tumorothèques. Ces collections de grande qualité, en particulier du fait des données cliniques qui y étaient associées, ont été utilisées dans des études de génomique somatique. Les études de transcriptomique réalisées avec différentes collections américaines $[8,9]$, espagnoles [10] et françaises [11, 12] ont révélé qu'après une classification non supervisée, chaque PGL/PCC ségrégeait selon son génotype dans l'un de deux clusters qui ont été définis. Le cluster 1 contenait les tumeurs SDHx (cluster 1A) et VHL (cluster 1B) alors que le cluster 2 regroupait les tumeurs liées aux gènes RET et NFI (Figure I). Le décryptage moléculaire de ces différents groupes a montré que chaque génotype était associé à une signature moléculaire spécifique. Par ailleurs, la classification dans l'un de ces groupes de tumeurs apparemment sporadiques ou familiales, mais d'origine génétique inconnue, a permis l'identification récente de nouveaux gènes du PGL/PCC, tels que TMEM127 (transmembrane protein 127) [13], SDHA [14] et MAX (MYC associated factor X) [15], ainsi qu'à la découverte de mutations somatiques dans les gènes RET et VHL [11]. Cette explosion des connaissances a apporté de nouvelles informations importantes pour le conseil génétique des patients présentant un PGL/PCC.

\section{Les principaux gènes de prédisposition aux PGL/PCC}

\section{Cluster $1 \mathrm{~A}$ : mutations de SDHD, SDHC, SDHB}

La succinate déshydrogénase (SDH) - ou complexe II mitochondrial - est composée de deux sous-unités catalytiques, SDHA et SDHB et de deux sous-unités d'ancrage, SDHD et SDHC. Son rôle dans le PGL/PCC héréditaire a été découvert il y a plus de dix ans, lors de l'identification des premières mutations de SDHD par des analyses de liaison génétique menées dans des familles dont certains membres étaient atteints de paragangliome héréditaire. Les mutations de SDHC et $S D H B$ ont ensuite été découvertes par des approches classiques de gène candidat. Le génotypage des trois gènes $S D H x$ a été réalisé à partir de différentes cohortes de patients, et les mutations identifiées à ce jour ont été collectées dans la TCA (tricarboxylic acid or Krebs cycle) gene mutation database (voir http:// chromium.liacs.nl/lovd_sdh/). Les PGL/PCC associés à des mutations de SDHD sont généralement multiples, souvent localisés dans la tête et le cou, et rarement malins (Tableau 1). Par ailleurs, le gène SDHD étant soumis à empreinte maternelle, une histoire familiale de PGL/PCC dans la branche paternelle devrait 


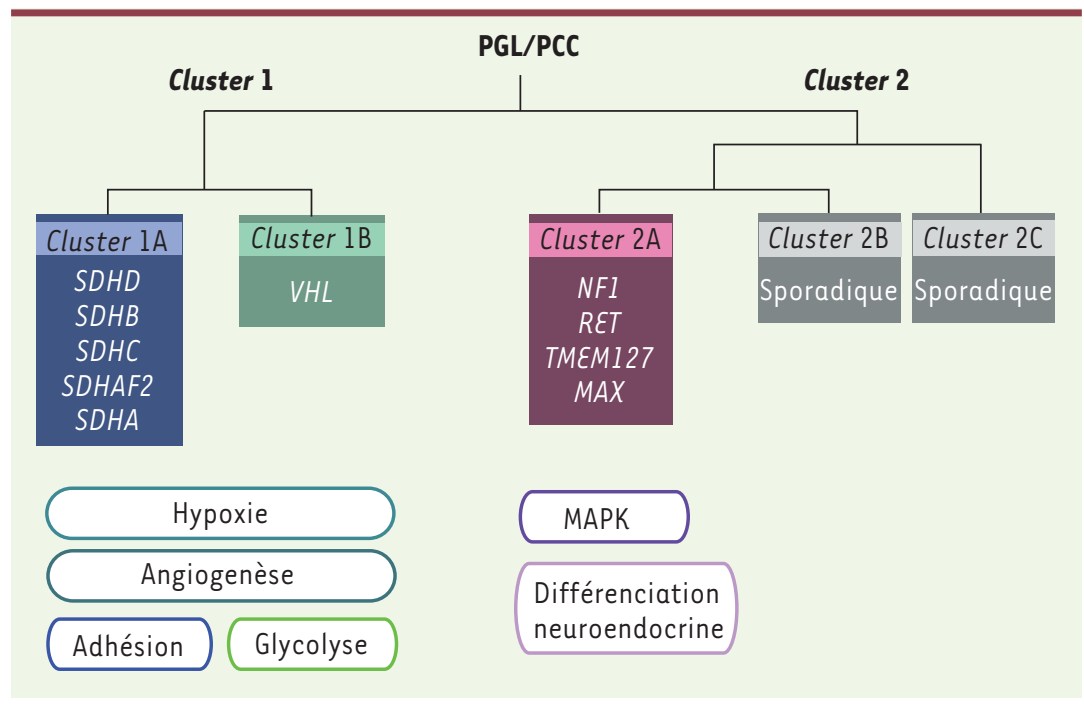

Figure 1. Représentation schématique de la classification non supervisée des PGL/PCC basée sur le transcriptome. Le cluster 1 contient toutes les tumeurs porteuses d'une mutation d'un gène $S D H x$ d'une part ( $1 \mathrm{~A})$ et $V H L$ d'autre part (1B). Ces tumeurs présentent une signature transcriptomique commune d'activation de la réponse hypoxique et de l'angiogenèse, mais aussi des particularités comme un profil d'adhésion pour les tumeurs ayant des mutations de SDHx et une augmentation de la glycolyse pour celles porteuses de mutations VHL. Les tumeurs porteuses de mutations NFI, RET, TMEM127 et MAX forment dans le cluster 2, avec la majorité des tumeurs sporadiques, et sont notamment associées à l'activation de la voie des MAPkinases et à un profil de différenciation neuroendocrine (adapté de [15]).

orienter le diagnostic génétique vers la recherche d'une mutation dans ce gène. La pathologie associée à des mutations de SDHB se présente, quant à elle, le plus souvent sous la forme d'une tumeur unique. II a été établi que l'identification d'une mutation SDHB est un facteur de risque de malignité et de mauvais pronostic. Les PGL/ PCC dépendants de SDHC sont, pour des raisons inconnues, plus rarement décrits. Mais la pénétrance de la maladie est importante chez les porteurs d'une mutation $S D H C$, qui développent une maladie similaire à celle des sujets SDHD.

Le diagnostic génétique des gènes $S D H x$ peut être guidé par l'immunohistochimie de la protéine SDHB dans le tissu tumoral après son exérèse chirurgicale. Ainsi, il a été démontré qu'un immunomarquage négatif pour la protéine SDHB est associé à la présence d'une mutation dans l'un des gènes $S D H x$ [16]. Le cluster l, qui englobe les tumeurs impliquant le gène $S D H$, mais aussi VHL, est caractérisé par une activation anormale de la voie de la réponse à l'hypoxie (processus dit de pseudohypoxie). En bref, pour les tumeurs SDH, il a été démontré que l'inactivation de la succinate déshydrogénase conduit à l'accumulation de succinate qui agit comme un inhibiteur compétitif des prolyl-hydroxylases, enzymes dont l'activité conduit à la dégradation des hypoxia-inducible factors (HIF) en conditions normoxiques [17]. Ainsi, même en présence d'oxygène, les protéines HIF non hydroxylées ne peuvent être reconnues par la protéine pVHL qui induit normalement leur ubiquitinylation et leur dégradation subséquente dans le protéasome (Figure 2). Elles sont alors stabilisées et activées et induisent notamment l'angiogenèse (pour revue, voir [18]). Cette caractéristique justifie l'utilisation possible des thérapies antiangiogéniques dans la prise en charge des PGL/PCC malins, qui sont souvent liés à une mutation SDHB [19].

\section{Cluster 1B : mutations de VHL}

La maladie de von Hippel-Lindau (VHL), caractérisée notamment par le développement de carcinomes du rein à cellules claires, d'hémangioblastomes du système nerveux central ou de la rétine, de tumeurs pancréatiques et de PGL/PCC, est causée par des mutations inactivatrices du gène suppresseur de tumeur VHL (pour revue, voir [20]). Le produit de ce gène est une ubiquitine ligase $\varepsilon 3$ dont l'activité en normoxie conduit à la dégradation des HIF par le protéasome, ce qui explique le profil pseudohypoxique (cluster 1 ) des tumeurs qui ont perdu la fonction VHL (Figure 2). Les PGL/PCC liés à des mutations VHL sont souvent bilatéraux ou multiples, rarement malins et de survenue plus précoce que dans d'autres syndromes héréditaires (Tableau I). En conséquence, les mutations VHL sont détectées chez plus de $40 \%$ des patients pédiatriques porteurs d'un PGL/PCC [21]. Par ailleurs, des mutations somatiques de VHL associées à la perte du chromosome $3 p$ ont été découvertes dans des PGL/PCC sans mutation germinale de VHL, mais dont le profil d'expression génique les classait dans le cluster 1B [11]. Le risque de développer un PCC et/ ou un carcinome rénal est à la base des associations génotype/phénotype de la maladie de VHL. Ainsi, le sous-type 2 définit les patients à haut risque de PGL/ PCC, alors que dans le VHL de type 1 , le risque est faible de développer un PCC, mais élevé de développer un carcinome rénal. Cette pénétrance phénotypique variable semble résulter d'une exigence, par les cellules chromaffines ou leurs précurseurs, d'une fonction résiduelle de VHL qui serait superflue pour les cellules rénales. Il est ainsi extrêmement rare de trouver des délétions complètes ou des mutations tronquantes du gène VHL dans les PGL/PCC, alors qu'elles sont fréquentes dans les formes de la maladie associées au cancer du rein. II a été proposé que les mutations faux-sens du gène, responsables de la maladie de type $2 C$ (qui ne conduit qu'à des PGL/PCC), affecteraient des fonctions de VHL indépendantes des HIF et perturberaient la voie apoptotique dépendante de Jun, favorisant ainsi la croissance tumorale. 


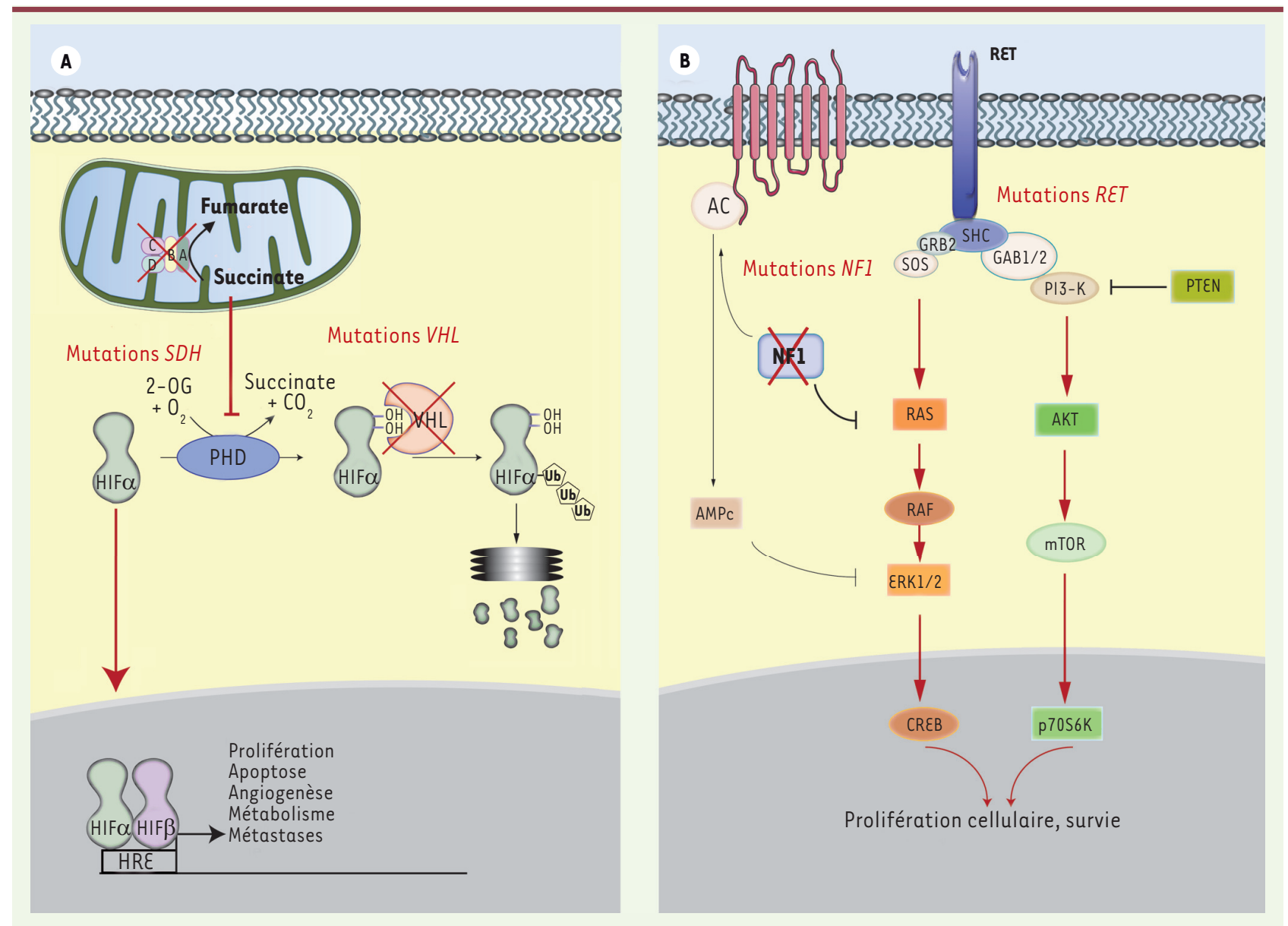

Figure 2. Mécanismes moléculaires de tumorigenèse dans les phéochromocytomes des clusters 1 et 2. Dans les tumeurs du cluster 1 (A), l'accumulation de succinate ou l'inactivation de VHL conduit à la stabilisation et à l'activation des HIF, même en présence d'oxygène. Dans les tumeurs du cluster 2 (B), l'activation du récepteur RET ou la perte de la fonction NFl est responsable d'une activation des voies RAS/RAF et AKT/mTOR. $\mathrm{Ub}$ : ubiquitine; AC : adénylate cyclase; AMPc : AMP cyclique; SHC : Src homology 2 domain containing protein ; GRB2 : growth factor receptorbound protein 2 ; SOS : son of sevenless protein; PTEN : phosphatase and tensin homolog protein ; CREB : cAMP response element-binding ; 2-OG : 2-oxoglutarate; PHD : prolyl hydroxylose; TOR : target of rapamycin.

\section{Cluster 2A : mutations de RET}

Les mutations constitutionnelles activatrices du gène codant pour le récepteur à activité tyrosine kinase RET sont responsables de la prédisposition à la néoplasie endocrinienne multiple de type 2 (NEM 2) qui comprend deux sous-types : la NEM 2A, caractérisée par la présence d'un PCC, d'un carcinome médullaire de la thyroïde (CMT) et d'une hyperparathyroïdie; et la $N E M$ 2B, le sous-type le plus agressif cliniquement, qui inclut PCC, CMT, ganglioneuromes et anomalies squelettiques; et, d'autre part, le CMT familial (FCMT), maladie dans laquelle les tumeurs de la thyroïde constituent la caractéristique clinique unique. RET est l'unique proto-oncogène parmi les gènes de susceptibilité au PGL/PCC. Les mutations du gène RET sont regroupées autour de points chauds dans certains exons ou codons spécifiques qui déterminent le degré de modification de l'activité du récepteur mutant : les mutations affectant les exons $10,11,13$ et 14 sont liées à la NEM 2A et au FCMT, tandis que celles des exons 15 et 16 sont détectées chez les patients NEM 2B. Cette relation génotype/phénotype particulière a permis d'établir un classement des mutations en fonction du risque d'agressivité de la maladie [22]. L'impact du dépistage des mutations du gène RET sur la gestion clinique et l'évolution naturelle de la NEM 2 représente l'un des exemples les plus anciens et les plus aboutis de soins personnalisés orientés par la génétique, une démarche dont on peut penser qu'elle constituera l'avenir de la pratique médicale. Le PCC est rarement la première manifestation de la NEM 2, et la proportion de mutations RET dans les PCC apparemment non syndromiques est plus faible (environ $5 \%$ ) que dans les autres syndromes. Les principales caractéristiques des PCC associés à RET sont leur bilatéralité et la rareté des formes malignes ou extrasurrénaliennes (Tableau 1). Ces tumeurs sont caractérisées par une activation des voies de signalisation intracellulaires phosphoinositide 3-kinase (PI3K)/protéine kinase B (ou 
AKT) et MAPK/extracellular signal-regulated kinases (ERK) (Figure 2) dont les cibles sont significativement enrichies dans le cluster $2[8,10$, 11]. Comme dans le cas de VHL, des mutations somatiques de RET ont été trouvées dans des tumeurs sporadiques classées dans le cluster 2, confirmant ainsi que des mutations somatiques peuvent aussi être responsables d'un nombre important de tumeurs (14,3\% des PGL/PCC de la collection du réseau français COMETE) [11].

\section{Cluster 2A : mutations de NF1}

La neurofibromatose de type 1 (ou maladie de von Recklinghausen) est une maladie héréditaire fréquente, à forte pénétrance, caractérisée par des neurofibromes multiples, des taches café au lait, des lentigines axillaires ou inguinales ${ }^{1}$, des anomalies squelettiques et une prédisposition aux leucémies et aux tumeurs gliales [23]. Le PCC y survient dans 0,1 à $5,7 \%$ des cas. La maladie se développe en raison de mutations du gène suppresseur de tumeur NFl, qui code pour une GTPase activating protein (GAP) impliquée dans la voie RAS (Figure 2). Les patients atteints de neurofibromatose présentent des PCC uni- ou bilatéraux, avec une incidence de tumeurs malignes légèrement supérieure à ce qu'elle est dans les cas liés à $V H L$ ou à RET. Le spectre des mutations NFI chez les patients atteints de PCC n'a pas été établi de manière très approfondie, car le diagnostic est basé sur les paramètres cliniques et ne fait pas l'objet d'un dépistage génétique de routine. Les résultats disponibles dans la littérature, basés sur l'analyse d'une seule cohorte de PCC, montrent que les mutations NFI semblent être majoritairement situées dans le domaine cystéine-sérine du gène (dans environ un tiers des cas), tandis que les mutations du domaine RAS-GAP - qui représente la principale cible fonctionnelle des mutations dans la neurofibromatose de type 1 - ne sont retrouvées que dans $13 \%$ des cas de PCC [24]. L'augmentation de l'activation de RAS et de la signalisation mTOR (target of rapamycin) explique le regroupement des PCC mutés sur NFI avec les tumeurs RET au sein du cluster 2 .

\section{Les nouveaux gènes de prédisposition aux PGL/PCC}

\section{Cluster 1A : mutations de SDHA et SDHAF2}

Alors que les études transcriptomiques ont permis de regrouper, élégamment et de façon non supervisée, les PGL/PCC génétiquement déterminés entre eux selon le gène impliqué, les tumeurs pour lesquelles aucune mutation n'avait été identifiée se sont réparties au sein de l'un de ces deux groupes spécifiques. Ce fut le cas du PGL extrasurrénalien d'une patiente chez laquelle aucune mutation des gènes $S D H D, S D H C$ ou SDHB n'était identifiée et dont le cas a récemment été rapporté par Burnichon et al. [14]. Les études réalisées sur cette tumeur ont montré l'activation de la réponse hypoxique ainsi que l'inhibition de l'activité enzymatique de la succinate déshydrogénase associée à la perte d'expression de SDHB et SDHA en immunohistochimie. Ces données suggéraient une anomalie du gène SDHA et ont abouti à l'identification de la première mutation constitutionnelle

${ }^{1}$ Taches rondes et planes, allant du brun au noir. de SDHA dans un contexte de PGL/PCC. Par la suite, un dépistage de l'expression de SDHA a été réalisé par immunohistochimie sur une série de 316 PGL/PCC et a conduit à l'identification d'une mutation SDHA chez cinq nouveaux sujets [25]. Bien que ce gène soit probablement un gène mineur dans la prédisposition au PGL/ $P C C$, son génotypage doit donc désormais être envisagé chez les patients chez lesquels l'analyse immunohistochimique du tissu tumoral révèle l'absence d'expression des protéines SDHB et SDHA.

Le gène SDHAF2 est un autre partenaire des gènes $S D H x$. II a été découvert grâce à l'identification de son orthologue SDH5 chez la levure. SDH5/SDHAF2 est impliqué dans la flavination de la protéine SDHA qui est nécessaire à l'assemblage du complexe II mitochondrial. Le gène SDHAF2 humain correspond au locus PGL2, identifié il y a plus de quinze ans dans une grande famille hollandaise atteinte de PGL de la tête et du cou [26]. Les premières données montrent que les mutations de SDHAF2 semblent associées à une symptomatologie souvent multifocale et à un jeune âge d'apparition de la maladie [27]. Toutefois, de nombreux sujets chez lesquels la mutation SDHAF2 a été détectée par le dépistage génétique semblaient asymptomatiques au moment du diagnostic, suggérant une pénétrance faible de la maladie [28]. Bien que la participation du gène SDHAF2 à la génétique des PGL/PCC semble relativement mineure, le génotypage de ce gène devrait donc être considéré chez un patient atteint de PGL de la tête et du cou, qu'il existe ou non une histoire familiale dans la branche paternelle, pour lequel un dépistage des gènes SDHD, SDHB et SDHC s'est avéré négatif, mais dont la tumeur n'exprime pas SDHB en immunohistochimie.

\section{Cluster 2A : mutations de TMEM127}

Le gène TMEM127, situé sur le chromosome $2 q 11$, code pour une protéine transmembranaire de 238 acides aminés qui ne présente ni motifs fonctionnels connus ni homologie avec une famille de protéines. Ce gène a été identifié en 2010 comme un gène de susceptibilité au PCC grâce à des approches de génomique intégrative, combinant analyse de liaison, étude du transcriptome et analyse du nombre de copies [13]. Les échantillons tumoraux provenant de patients avec une mutation TMEM127 se classent dans le cluster 2 ; il y a systématiquement une perte de l'allèle sauvage qui s'étend souvent sur la totalité du bras $2 q$ [13, 29, 30]. Les premières données obtenues in vitro suggèrent que TMEM127 pourrait être impliquée dans le trafic protéique intracellulaire et/ou dans le recyclage des protéines de l'endocytose. TMEM127 semble également jouer un rôle dans l'inhibition de la signalisation mTOR [13]. Les 


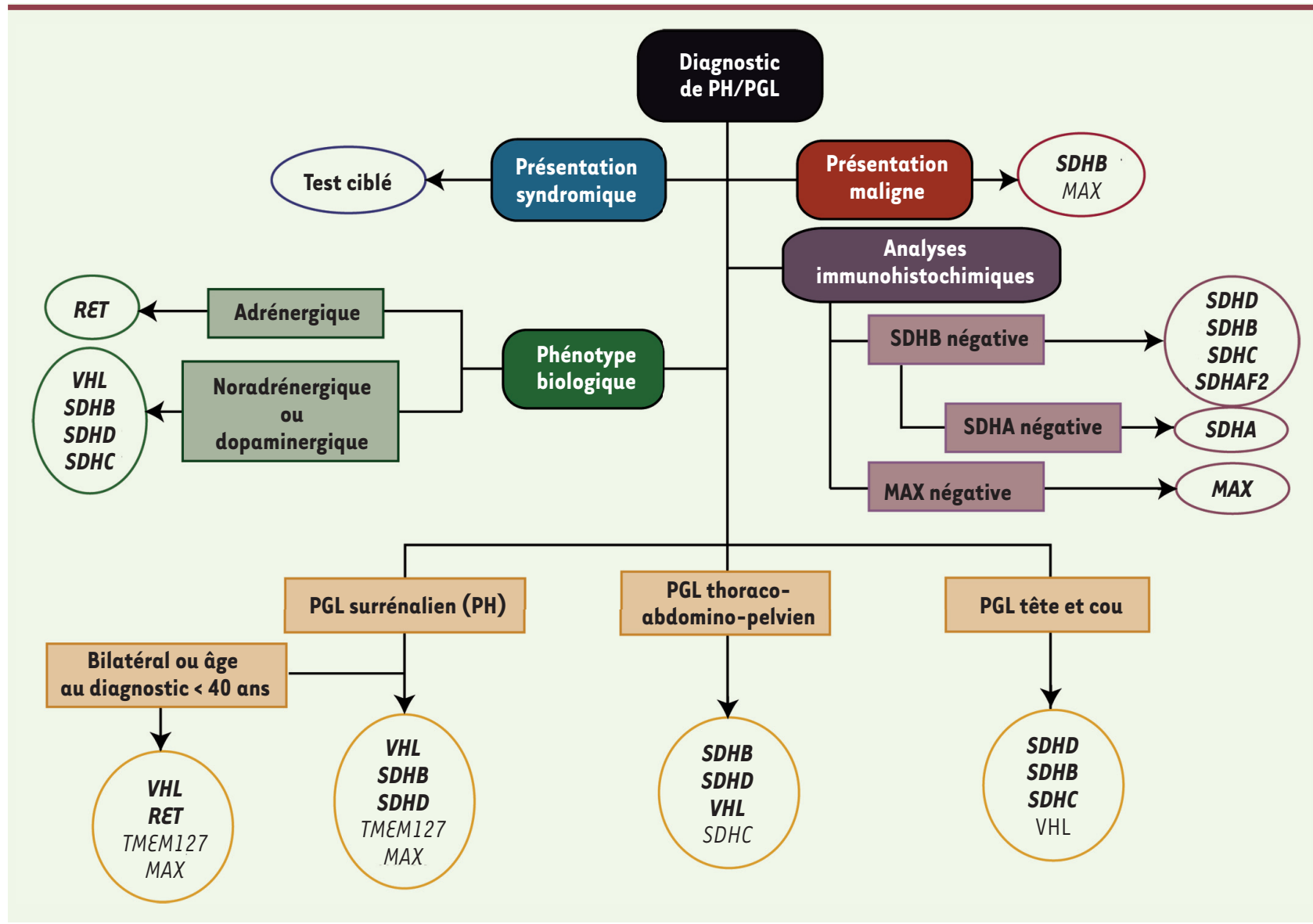

Figure 3. Arbre décisionnel pour le conseil génétique du PGL/PCC. La présentation clinique, le phénotype biologique et les analyses immunohistochimiques permettent de cibler le test génétique vers les gènes de prédisposition à analyser en première intention (adapté d'après les recommandations du réseau INCa des laboratoires d’oncogénétique des tumeurs endocrines, GTE-RENATEN, novembre/décembre 2011). Les gènes à tester en priorité sont indiqués en gras et ordonnés selon une priorité descendante (de haut en bas).

études génétiques indiquent que les patients porteurs de mutations TMEM127 représenteraient une faible proportion des patients atteints de PCC (environ $2 \%$ des cas sans autre mutation identifiée) et les premières associations génotype/phénotype montrent un âge moyen à la présentation (42 ans) similaire à celui des cas sporadiques, et plus élevé que chez la plupart des porteurs d'une mutation dans un gène de susceptibilité [30]. Toutes les mutations signalées à ce jour sont constitutionnelles et sont préférentiellement situées à l'intérieur ou autour des domaines transmembranaires de la protéine.

\section{Cluster 2A: mutations de MAX}

MAX (Myc-associated factor $\mathrm{X}$ ) est le gène de susceptibilité au PCC le plus récemment identifié. La découverte de ce $10^{e}$ gène de prédisposition au PCC a été rendue possible par le séquençage nouvelle génération de l'ensemble des exons (exome) dans trois familles indépendantes présentant des cas de PCC, mais exemptes de mutation de l'un des gènes de prédisposition au PGL/PCC [15]. Ces tumeurs avaient en commun une signature moléculaire robuste et distinctive, les incluant dans le cluster 2. La fonctionnalité des mutations MAX a été validée par la démonstration de la perte de l'allèle sauvage (perte d'hétérozygotie $[\mathrm{LOH}]$ ) et de l'absence d'expression de la protéine MAX dans ces tumeurs. MAX est un membre clé de la famille des facteurs de transcription MYC/ MAX/MXDI (MAX dimerization protein-1) qui régule la croissance cellulaire, la prolifération et la biologie des cellules souches au cours du développement normal et de l'homéostasie tissulaire [31]. MAX peut former des homodimères, ainsi que des hétérodimères avec d'autres protéines de cette voie, et il agit comme un répresseur transcriptionnel. II se comporte donc comme un antagoniste de la transformation cellulaire dépendante de MYC. De façon intéressante, il a été montré que les cellules issues de $\mathrm{PCC}$ de la lignée de rat $\mathrm{PCl} 2$ portent une mutation homozygote de Max qui inactive sa fonction. Après la découverte que MAX était un nouveau gène de susceptibilité au PCC, une recherche de mutations a été réalisée chez 59 patients présentant un PCC bilatéral et/ou chez lesquels la tumeur était apparue avant trente ans. Cette étude a permis la détection des mutations de ce gène chez cinq patients supplé- 
mentaires ( $13 \%$ des patients de cette série soigneusement sélectionnée), dont plus de la moitié avaient un PCC bilatéral [15]. De façon intéressante, une transmission préférentielle des mutations MAX par la branche paternelle a été trouvée chez les porteurs touchés. Si cette observation est confirmée, il s'agirait, après SDHD et SDHAF2 [5, 26], du $3^{e}$ exemple de transmission liée au sexe dans le PCC héréditaire. Ce mode de transmission pourrait rendre plus difficile l'identification de cas de PCC familiaux si la mutation a été transmise par les mères sur plusieurs générations, et expliquer l'absence d'histoire familiale de PCC retrouvée chez quelques patients MAX.

\section{Conclusions}

Depuis dix ans, la complexité croissante des connaissances de la génétique des $P G L / P C C$ a conduit à une remise à jour régulière des différents algorithmes du conseil génétique appliqué à ces patients (Figure 3). Un dépistage génétique rationnel devrait désormais être basé sur la combinaison de l'examen clinique détaillé et de l'enquête familiale, de l'analyse minutieuse des données biochimiques, et de l'évaluation immunohistochimique précise du tissu tumoral après son exérèse chirurgicale. Avec l'apparition des nouvelles techniques de séquençage et la diminution tout aussi rapide des coûts de ces nouvelles technologies, les méthodes de séquençage nouvelle génération (next generation sequencing ou NGS) vont probablement remplacer les techniques classiques de diagnostic des mutations. Ainsi, dans un futur proche, une procédure ciblée par étapes telle qu'elle se pratique aujourd'hui pour le test génétique du PGL/PCC sera probablement obsolète. De nouvelles stratégies devront alors être mises en œuvre pour faire face à un grand nombre de nouveaux variants de signification inconnue qui seront probablement identifiés par ces nouvelles méthodes. Le NGS pourrait aussi aider à détecter des mutations rares qui n'ont pas été diagnostiquées par les outils moléculaires utilisés en routine. En effet, malgré l'intensité des recherches menées avec succès au cours de la dernière décennie, le dépistage reste négatif chez une proportion non négligeable de patients (jusqu'à $10 \%$ ), alors qu'ils sont vraisemblablement porteurs d'une mutation dans un gène de prédisposition au PGL/PCC. L'utilisation et l'intégration des données génomiques issues de l'analyse du transcriptome, du miRnome, de la cartographie détaillée des pertes et des gains chromosomiques par SNP (single nucleotide polymorphism) array et/ou du statut de méthylation de I'ADN permettront sûrement aussi de découvrir d'autres gènes de susceptibilité au PGL/PCC. Les années futures permettront sans doute une prise en charge personnalisée des patients, chez qui le suivi et les stratégies thérapeutiques pourront être adaptés (à la carte), à leur statut moléculaire constitutionnel et/ou somatique. $\diamond$

\section{SUMMARY}

Genetics of paragangliomas and pheochromocytomas

Pheochromocytomas and paragangliomas are rare tumors that arise from chromaffin tissues of the adrenal medulla and from sympathetic and parasympathetic ganglia. Ten susceptibility genes (VHL, RET, NFl, SDHA, B, C and D, SDHAF2, TMEM127 and finally MAX) are responsible for at least $30 \%$ of the cases. These patients can either have a familial history of the disease, a syndromic presentation, but can also emerge as apparently sporadic forms. Moreover, recent transcriptomic studies have led to the identification of somatic mutations in VHL and RET genes in $15 \%$ of sporadic cases, thus bringing a molecular explanation for nearly half of all cases. These findings have had a major impact on the understanding of the molecular mechanisms of tumorigenesis in pheochromocytomas and paragangliomas. It was shown that the genetic status of tumors is associated with particular transcription signatures, such as the activation of the pseudohypoxic response or the activation of MAPK or mTOR signalling. From nowadays to a close future, these advances will have important consequences on the monitoring of patients, from genetic counseling to a personalized clinical management. $\diamond$

\section{LIENS D'INTÉRÊT}

Les auteurs déclarent n'avoir aucun lien d'intérêt concernant les données publiées dans cet article.

\section{RÉFÉRENCES}

1. Lenders JW, Eisenhofer G, Mannelli M, Pacak K. Phaeochromocytoma. Lancet $2005 ; 366: 665-75$.

2. Wallace MR, Marchuk DA, Andersen LB, et al. Type 1 neurofibromatosis gene: identification of a large transcript disrupted in three NFl patients. Science $1990 ; 249$ : 181-6.

3. Mulligan LM, Kwok JB, Healey CS, et al. Germ-line mutations of the RET proto-oncogene in multiple endocrine neoplasia type 2A. Nature 1993; 363 : 458-60.

4. Gnarra JR, Tory K, Weng $Y$, et al. Mutations of the VHL tumour suppressor gene in renal carcinoma. Nat Genet $1994 ; 7: 85-90$.

5. Baysal BE, Ferrell RE, Willett-Brozick JE, et al. Mutations in SDHD, a mitochondrial complex II gene, in hereditary paraganglioma. Science $2000 ; 287: 848-51$.

6. Niemann S, Muller U. Mutations in SDHC cause autosomal dominant paraganglioma, type 3. Nat Genet $2000 ; 26: 268-70$.

7. Astuti D, Latif F, Dallol A, et al. Gene mutations in the succinate dehydrogenase subunit SDHB cause susceptibility to familial pheochromocytoma and to familial paraganglioma. Am J Hum Genet $2001 ; 69: 49-54$.

8. Dahia PL, Ross KN, Wright ME, et al. A HIFlalpha regulatory loop links hypoxia and mitochondrial signals in pheochromocytomas. PLoS Genet $2005 ; 1: 72-80$

9. Eisenhofer G, Huynh $\Pi$, Pacak K, et al. Distinct gene expression profiles in norepinephrine- and epinephrine-producing hereditary and sporadic pheochromocytomas: activation of hypoxia-driven angiogenic pathways in von Hippel-Lindau syndrome. Endocr Relat Cancer 2004 ; 11 : 897-911.

10. Lopez-Jimenez $\varepsilon$, Gomez-Lopez G, Leandro-Garcia LJ, et al. Research resource: transcriptional profiling reveals different pseudohypoxic signatures in SDHB and VHL-related pheochromocytomas. Mol Endocrinol $2010 ; 24: 2382-91$.

11. Burnichon N, Vescovo L, Amar L, et al. Integrative genomic analysis reveals somatic mutations in pheochromocytoma and paraganglioma. Hum Mol Genet $2011 ; 20: 3974-85$.

12. Favier J, Briere JJ, Burnichon $\mathrm{N}$, et al. The warburg effect is genetically determined in inherited pheochromocytomas. PloS One 2009; 4 : e7094.

13. Qin $Y$, Yao L, King $\varepsilon \varepsilon$, et al. Germline mutations in TMEM127 confer susceptibility to pheochromocytoma. Nat Genet $2010 ; 42: 229-33$.

14. Burnichon N, Briere JJ, Libe R, et al. SDHA is a tumor suppressor gene causing paraganglioma. Hum Mol Genet $2010 ; 19$ : 3011-20.

15. Comino-Mendez I, Gracia-Aznarez FJ, Schiavi F, et al. Exome sequencing identifies MAX mutations as a cause of hereditary pheochromocytoma. Nat Genet $2011 ; 43: 663-7$. 


\section{RÉFÉRENCES}

16. Van Nederveen FH, Gaal J, Favier J, et al. An immunohistochemical procedure to detect patients with paraganglioma and phaeochromocytoma with germline SDHB, SDHC, or SDHD gene mutations: a retrospective and prospective analysis. Lancet Oncol $2009 ; 10: 764-71$.

17. Pouyssegur J, Gothie $\varepsilon$. HIF-1 : régulateur central de l'hypoxie. Med Sci (Paris) $2002 ; 18: 70-8$.

18. Favier J, Gimenez-Roqueplo AP. Pheochromocytomas: the (pseudo)-hypoxic hypothesis. Best Pract Res Clin Endocrinol Metab 2010 ; 24 : 957-68.

19. Favier J, Igaz $\mathrm{P}$, Burnichon $\mathrm{N}$, et al. Rationale for anti-angiogenic therapy in pheochromocytoma and paraganglioma. Endocr Pathol $2011 ; 23: 34-42$.

20. Barontini M, Dahia PL. VHL disease. Best Pract Res Clin Endocrinol Metab 2010 ; 24 : 401-13.

21. Neumann HP, Bausch B, McWhinney SR, et al. Germ-line mutations in nonsyndromic pheochromocytoma. N Engl J Med 2002 ; 346 : 1459-66.

22. Kloos RT, Eng C, Evans DB, et al. Medullary thyroid cancer: management guidelines of the American thyroid association. Thyroid $2009 ; 19: 565-612$.

23. Wolkenstein P. La neurofibromatose 1. Med Sci (Paris) 2001 ; 17 : 1158-67.

24. Bausch B, Koschker AC, Fassnacht $M$, et al. Comprehensive mutation scanning of NFl in apparently sporadic cases of pheochromocytoma. J Clin Endocrinol Metab 2006 ; 91 : 3478-81.

25. Korpershoek $\varepsilon$, Favier J, Gaal J, et al. SDHA immunohistochemistry detects germline SDHA gene mutations in apparently sporadic paragangliomas and pheochromocytomas. J Clin Endocrinol Metab $2011 ; 96: \varepsilon 1472-6$.

26. Hao HX, Khalimonchuk 0 , Schraders $M$, et al. SDH5, a gene required for flavination of succinate dehydrogenase, is mutated in paraganglioma. Science 2009 ; $325: 1139-42$
27. Bayley JP, Kunst HP, Cascon A, et al. SDHAF2 mutations in familial and sporadic paraganglioma and pheochromocytoma. Lancet Oncol 2010 ; $11: 366-72$.

28. Kunst HP, Rutten MH, de Monnink JP, et al. SDHAF2 (PGL2-SDH5) and hereditary head and neck paraganglioma. Clin Cancer Res $2011 ; 17$ : 247-54.

29. Burnichon N, Lepoutre-Lussey C, Laffaire J, et al. A novel TMEM127 mutation in a patient with familial bilateral pheochromocytoma. Eur J Endocrinol $2011 ; 164: 141-5$.

30. Yao L, Schiavi F, Cascon A, et al. Spectrum and prevalence of FP/TMEM127 gene mutations in pheochromocytomas and paragangliomas. JAMA 2010 $304: 2611-9$

31. Grandori C, Cowley SM, James LP, Eisenman RN. The Myc/Max/Mad network and the transcriptional control of cell behavior. Annu Rev Cell Dev Biol $2000 ; 16: 653-99$.

\section{Collection SCIENCE ET BIOMÉDECINE}

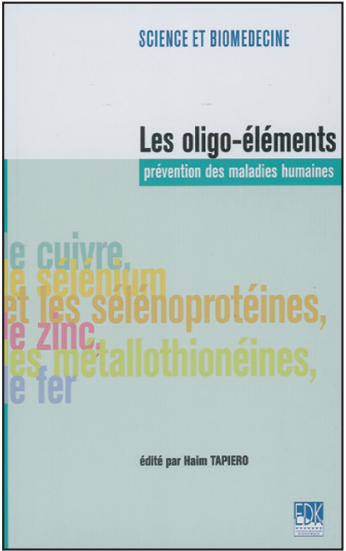

ISBN : 2-84254-107-3 64 pages

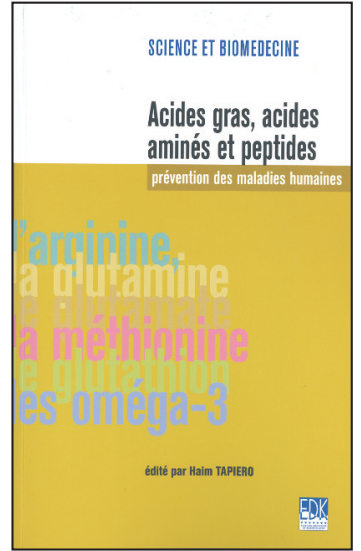

ISBN : 2-84254-108-1 80 pages

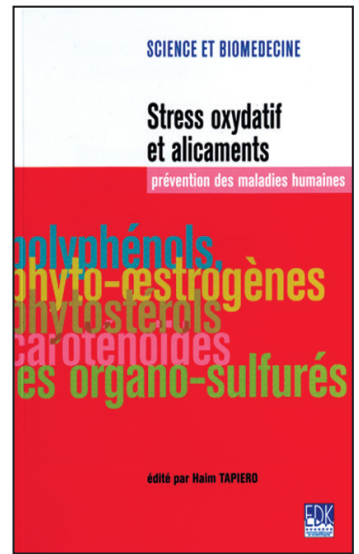

ISBN : 2-84254-111-1 86 pages

\section{Bon de commande}

À retourner à EDK, 25, rue Daviel - 75013 Paris

Tél. : 0158101905 - Fax : 0143293262 - E-mail : edk@edk.fr

NOM : Prénom :

Adresse :

Code postal :

Ville :

Pays :

Fonction :

Je souhaite recevoir l'ouvrage Les oligo-éléments : $10 €+3 €$ de port $=\mathbf{1 3} €$ TTC

Je souhaite recevoir l'ouvrage Acides gras, acides aminés et peptides : $12 €+3 €$ de port $=\mathbf{1 5} €$ TTC

Je souhaite recevoir l'ouvrage Stress oxydatif et alicaments : $14 €+3 €$ de port $=\mathbf{1 7} €$ TTC

$\square$ exemplaire, soit un total de $€$

Par carte bancaire :

$\square$ Visa $\square$ Eurocard/Mastercard

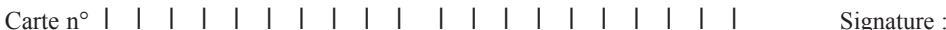

Date d'expiration :

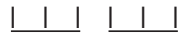

$N^{\circ}$ de contrôle au dos de la carte : $\quad$ | $1 \quad$ |

TIRÉS À PART

J. Favier 